\title{
Communication
}

\section{Apparition des chaleurs et de la décharge préovulatoire de LH chez la brebis de race Djallonké après synchronisation des chaleurs avec ou sans PMSG}

\author{
G. Toure ${ }^{1}$ \\ C. Meyer ${ }^{2}$ \\ A. Kouassi ${ }^{1}$
}

TOURE G., MEYER C., KOUASSI A. Apparition des chaleurs et de la décharge préovulatoire de LH chez la brebis de race Djallonké après synchronisation des chaleurs avec ou sans PMSG. Revue Elev. Méd. vét. Pays trop., 1995, 48 (4) : 357-361

En vue d'étudier l'influence de la PMSG sur l'apparition des chaleurs et le pic préovulatoire de $\mathrm{LH}$ chez les brebis Djallonké, les œestrus de 80 brebis cyclées ont été synchronisés à l'aide d'éponges vaginales d'acétate de fluorogestone en saison sèche et chaude. Ces brebis ont été réparties en deux lots ( $E$ et $P$ ) de même effectif. Au retrait des éponges le $14^{e}$ jour après la pose, chaque brebis du lot $\mathrm{P}$ a reçu une injection de 350 UI de PMSG alors que celles du lot E n'ont reçu aucun traitement. Toutes les deux heures, le comportement sexuel a été observé chez toutes les brebis à l'aide d'un bélier vasectomisé. Un jour après le retrait des éponges, des prélèvements sanguins ont été effectués toutes les 2 heures pendant 4 jours consécutifs chez 5 brebis dans chaque lot. Ces prélèvements sanguins ont été utilisés pour doser par RIA l'hormone lutéinisante (LH) Les brebis sont venues en chaleur plus précocement $(p<0,05)$ dans le lot $\mathrm{P}$ que dans le lot $\mathrm{E}(37,2 \pm 13,3 \mathrm{~h}$ contre $43,7 \pm 14,3 \mathrm{~h}$ pour le délai retrait des éponges - premiers signes de chaleur). Cependant la durée des chaleurs $(43,6 \pm 20,8 \mathrm{~h})$ est comparable dans les deux lots. La décharge préovulatoire de LH apparaît plus tôt dans le lot $\mathrm{P}$ que dans le lot $\mathrm{E}(12,4$ $\pm 1,5 \mathrm{~h}$ contre $19,5 \pm 7,7 \mathrm{~h}$ après le début de l'œstrus ; $p<0,05$ ). En revanche, la durée de ce pic de $\mathrm{LH}(10,7 \pm 4,1 \mathrm{~h})$ et sa valeur maximale $(90,5 \pm 36,4 \mathrm{ng} / \mathrm{ml})$ sont semblables dans les deux lots. Globalement, les caractéristiques de chaleur et de pic de LH chez la race Djallonké semblent proches de celles antérieurement décrites pour les races européennes. En outre, l'administration de PMSG hâte l'apparition des chaleurs et celle du pic de LH.

Mots-clés : Ovin - Brebis Djallonké - Synchronisation de l'œstrus - Cycle œstral - LH - Côte d'Ivoire.

1. Institut des Savanes (IDESSA), Département des Ressources animales, BP 633 Bouaké 01, Côte d'lvoire.

2. CIRAD-EMVT, BP 5035 - 34032 Montpellier Cedex 1, France.

Reçu le 3.11.93, accepté le 7.2.96.

\section{Introduction}

La production des élevages des petits ruminants est extrêmement dépendante des performances de reproduction des animaux ainsi que des relations qu'ils entretiennent avec leur environnement (3). Chez les femelles, l'amélioration des performances de reproduction nécessite la connaissance du cycle sexuel et en particulier de son complexe d'hormones dont les niveaux varient d'une part avec la race (10) et d'autre part avec le milieu (16, 20,22 ). En effet, pour contrôler la reproduction chez les ovins, il est important de maîtriser le moment d'ovulation afin de réaliser l'insémination artificielle ou la monte en main à un moment prédéterminé sans avoir nécessairement à détecter les chaleurs $(5,6)$. Cette maîtrise du moment d'ovulation nécessite la connaissance des phénomènes endocriniens régulateurs de la fonction sexuelle tels que les profils de l'hormone gonadotrophe LH d'origine hypophysaire et des stéroïdes gonadiques (oestrogènes, progestérone). Cette connaissance du système hormonal sexuel fait actuellement défaut en race Djallonké. II n'existe chez cette race que des données sur le profil de progestérone (13). Le présent travail se propose (chez la brebis Djallonké) de mesurer d'une part la durée de l'œestrus et d'autre part de déterminer l'évolution du niveau plasmatique de l'hormone' LH pendant les chaleurs. L'apparition des chaleurs a été synchronisée à l'aide d'un traitement progestagène à base d'acétate de fluorogestone appliqué sur une éponge vaginale. Ce traitement a été associé ou non à une injection de PMSG (Pregnant Mare's Serum Gonadotrophin) au retrait de l'éponge afin de connaitre ses effets sur le moment d'apparition du pic préovulatoire de LH pendant les chaleurs.

\section{Matériel et Méthode}

\section{Animaux}

L'essai a été réalisé sur 80 brebis Djallonké issues du troupeau ovin de l'Institut des Savanes (IDESSA) de Bouaké, Côte d'Ivoire. Ces brebis, âgées de 2 à 5 ans, ont été réparties en deux lots $E$ et $P$ de 40 têtes chacun. Les deux lots étaient composés de brebis de poids homogènes (lot $E=22,01 \pm 2,74 \mathrm{~kg}$; lot $P=22,06 \pm$ $2,83 \mathrm{~kg}$ ). Cinq béliers vasectomisés par lot de brebis ont été utilisés pour la détection des chaleurs. 
Tous ces animaux consommaient essentiellement du Panicum maximun issu de pâturage artificiel, coupé et distribué à volonté à la bergerie où les animaux étaient répartis à raison de 10 à 15 têtes par case avec de l'eau à volonté

\section{Méthode}

L'étude s'est déroulée en début de saison sèche et chaude. La température et l'humidité ambiante moyennes étaient respectivement de $26,5^{\circ} \mathrm{C}$ et 65,4 p. 100.

Des éponges vaginales (Chronogest@) de $40 \mathrm{mg}$ d'acétate de fluorogestone (FGA) ont été posées aux 80 brebis. Au retrait des éponges, le $14^{e}$ jour après leur pose, de la PMSG a été injectée par voie intramusculaire aux seules brebis du lot $\mathrm{P}$ à raison de $350 \mathrm{UI}$ par brebis. Les brebis $\mathrm{du}$ lot $E$ n'ont subi aucun traitement après le retrait des éponges.

L'observation des chaleurs pratiquée toutes les 2 heures pendant 4 jours consécutifs a commencé $24 \mathrm{~h}$ après le retrait des éponges. Elle se faisait sur toutes les brebis présentées en groupe de 10 à 15 têtes à un bélier vasectomisé qui permettait de repérer celles qui étaient en chaleur. Pour éviter l'effet de préférence sexuelle (19), la brebis détectée en chaleur était isolée du groupe.

Les prises de sang ont commencé $24 \mathrm{~h}$ après le retrait des éponges et se sont poursuivies toutes les deux heures pendant quatre jours consécutifs. Elles ont été effectuées seulement sur cinq brebis retenues au hasard dans chaque lot. Le sang a été prélevé à la veine jugulaire à l'aide de tubes héparinés. Ensuite, il a été centrifugé à 3000 tours $/ \mathrm{min}$ pendant $10 \mathrm{~min}$. Le plasma sanguin obtenu a été congélé à $-15^{\circ} \mathrm{C}$ en attendant d'être soumis au dosage de LH. Celui-ci a été effectué au moyen de la méthode radioimmunologique RIA (20).

Le pic de LH a été défini comme étant l'ensemble des valeurs dépassant le niveau de base de LH augmenté de 3 fois son écart-type. La durée du pic de LH n'a été déterminée que chez 4 brebis du lot $E$, une brebis de ce lot n'ayant pas terminé sa décharge préovulatoire de $\mathrm{LH}$ avant la fin de l'étude. Les délais d'apparition des chaleurs et du pic de LH ainsi que leurs durées respectives et la valeur maximale du pic de LH ont été comparés à l'aide du test $t$ de Student.

\section{Résultats}

L'apparition des chaleurs et du pic de LH sont indiqués dans le tableau I. L'évolution de la LH plasmatique et les périodes des chaleurs sont représentées dans la figure 1.

\section{Observation des chaleurs}

Au cours de la période des observations, 100 p. 100 des brebis ont été détectées en chaleur dans les 80 et $84 \mathrm{~h}$
I'ABLEAU I

Apparition des chaleurs et du pic de LH chez les brebis de race Djallonké

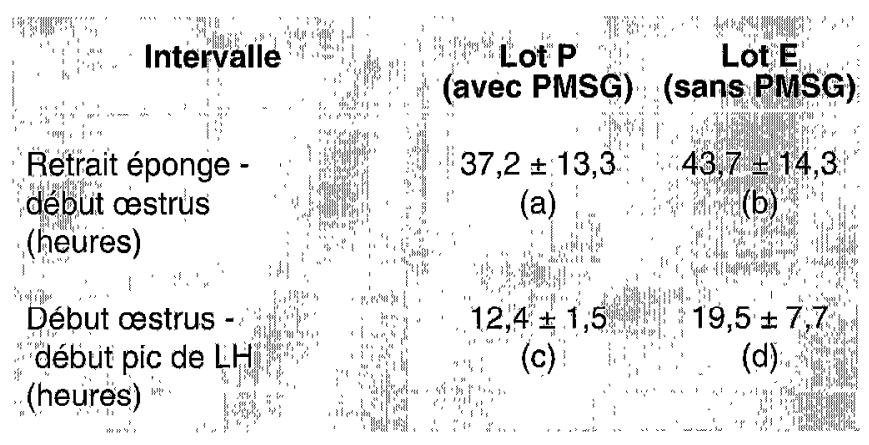

I es chiffres affectés de lettres différentes sont significativement différents au seuil de 5 p. $100 ; m \pm$ écart-type ; effectif $: n=5$ par lot

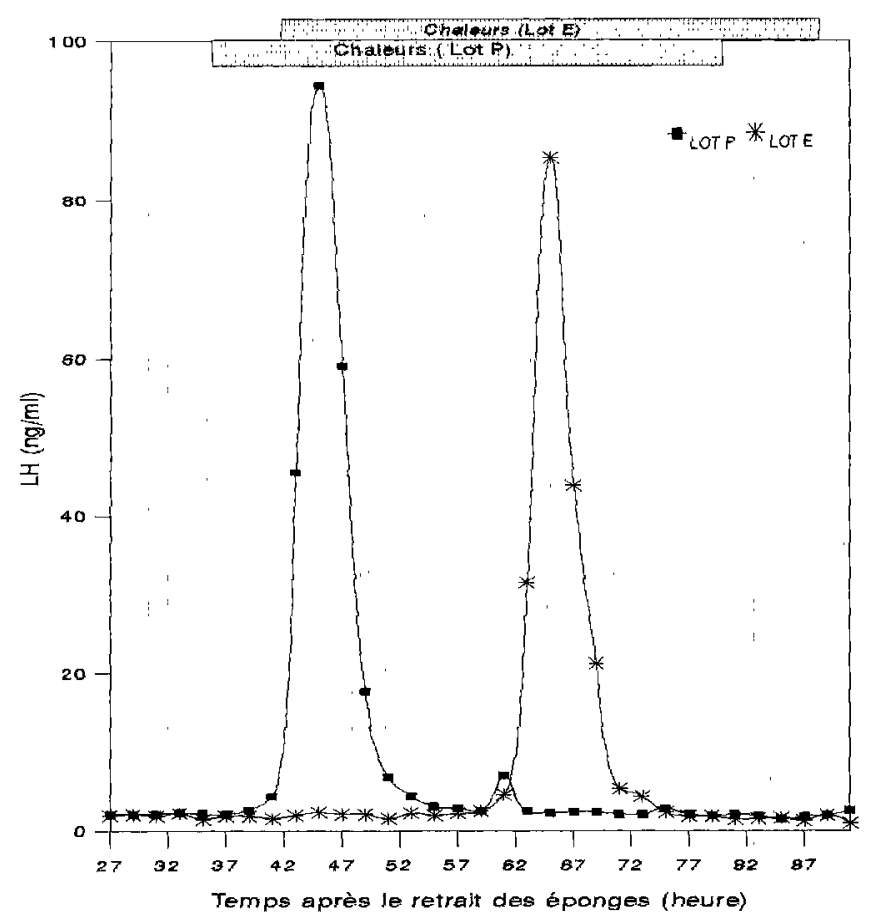

Figure 1 : Evolution de la LH plasmatique après le retrait des éponges chez la brebis Djallonké.

respectivement suivant le retrait des éponges dans le lot $P$ et le lot $E$. Le pourcentage cumulé des brebis on chaleurs (tableau II) montre que plus de la moitié des brebis ont été observées en œstrus $30 \mathrm{~h}$ et $44 \mathrm{~h}$ après le retrait des éponges respectivement dans le lot $P$ (à $P M S G$ ) et le lot E (sans PMSG).

Le délai moyen d'apparition des chaleurs après le retrait des éponges est significativement plus élevé $(p<0,05)$ 
TABLEAU II

Pourcentage cumulé des brebis en chaleur en fonction du (h) temps après le retrait des éponges

$\begin{aligned} & \text { Délai après le } \\ & \text { retrait des éponges } \\ & \text { (h) }\end{aligned}$
$\begin{aligned} & \text { Lot E } \\ & \text { (p. 100) }\end{aligned}$

dans le lot $E$ que dans le lot $P(43,7 \pm 14,3 \mathrm{~h}$ contre 37,2 $\pm 13,3 \mathrm{~h}$ ). Par ailleurs, il n'existe pas de différence significative ( $p>0,05$ ) entre les durées des chaleurs dans les deux lots dont la moyenne se situe à $43,6 \pm 20,8 \mathrm{~h}$.

II n'existe pas de différence statistiquement significative $(P>0,05)$ entre le délai d'apparition des chaleurs des 5 brebis ayant fait l'objet de dosage de LH et celles du lot dont elles sont issues. II en est de même de la durée des chaleurs. Elles sont bien représentatives de leur groupe.

\section{Niveau de LH}

Les brebis ayant fait l'objet de dosage de LH ont toutes présenté une décharge préovulatoire après le début des chaleurs. Le délai d'apparition du début de la décharge préovulatoire de LH après le début de l'œstrus est significativement plus élevé $(p<0,01)$ et avec un écart-type plus grand dans le lot $E$ que dans le lot $P(19,5 \pm 7.7 \mathrm{~h}$ contre $12,4 \pm 1,5 \mathrm{~h}$ ). La durée du pic de $\mathrm{LH}$ est identique dans les deux lots et est égale en moyenne à 10,7 \pm $4,1 \mathrm{~h}$.

Par ailleurs, le niveau de base moyen de LH est comparable dans les deux lots de brebis : 2,0 $\pm 0,6 \mathrm{ng} / \mathrm{ml}$. La valeur maximale du pic de LH est statistiquement semblable dans les deux lots et se situe à $90,5 \pm 36,4 \mathrm{ng} / \mathrm{ml}$ en moyenne.

\section{Discussion}

Le traitement de synchronisation des œstrus a été réalisé avec succès puisque toutes les brebis sont venues en chaleur au cours de l'étude. Avec un traitement hormonal de même type, Quirke (16) a obtenu une efficacité de synchronisation voisine de 100 p. 100 chez les brebis de races Galway et métisses. Au Nigeria, chez la même race que celle de la présente étude, Oyediji et al. (14) ont abouti à un résultat comparable en synchronisant à l'aide de progestagènes en implants sous-cutanés ou d'éponges vaginales

La différence de $7 \mathrm{~h}$ environ notée entre les délais d'apparition des chaleurs chez les' brebis des deux lots montre que la PMSG accélère la venue en chaleur des brebis traitées comme cela a déjà été constaté par Signoret et Cognie (18) chez la brebis lle-de-France. Un résultat comparable a été obtenu par Cognie et al. (4) qui trouvent que l'intervalle de temps entre le retrait des éponges et le début de l'œstrus est plus court chez les brebis ayant reçu de la PMSG que chez celles ayant eu des éponges seules $(31,4 \pm 0,56 \mathrm{~h}$ contre $39,9 \pm 0,90 \mathrm{~h})$. Le délai moyen d'apparition des chaleurs $(37,2 \pm 13,3 \mathrm{~h})$ obtenu chez les brebis du lot $P$ est comparable à la valeur de $36,7 \pm 1,3 \mathrm{~h}$ obtenue chez les brebis de race Karagoumico soumises au même type de traitement hormonal par Acritopoulo-Fourcroy et al. (1).

Chez la brebis Djallonké, la PMSG n'influence pas la durée des chaleurs. De même, les brebis soumises à trois traitements de synchronisation à base de prostaglandines $F 2 \alpha$ et de progestagènes sous forme d'implants sous-cutanés ou d'éponges vaginales présentent une même durée d'œstrus (14). La durée de l'œstrus est un caractère qui présente d'importantes variations selon les races des brebis (12). En effet, la durée des chaleurs est généralement longue chez les races hautement prolifiques (11). Chez la race Djallonké, Berger (2) a obtenu une durée d'œstrus variant de 12 à $60 \mathrm{~h}$, intervalle qui intègre notre valeur moyenne.

Les décharges préovulatoires de $\mathrm{LH}$ chez toutes les brebis dans les deux lots ont commencé après le début des 
chaleurs, confirmant ainsi les résultats de Thimonier et Pelletier (23). Ces décharges préovulatoires sont plus précoces chez les brebis ayant reçu de la PMSG que chez celles ayant eu des éponges seules. Ce résultat est en accord avec celui obtenu par Signoret et Cognie (18) qui constatent que l'injection de la PMSG accélère l'apparition du pic de LH. Cette différence de 7 heures est proche de celle observée entre les débuts de chaleurs des deux groupes; le traitement au PMSG n'influence pas l'intervalle début de l'œstrus-pic de LH. L'intervalle trouvé entre le début de l'œstrus et la décharge préovulatoire de LH chez la brebis Djallonké est différent de celui trouvé chez la brebis Romanov par Thimonier (21) qui s'élève à $17,6 \mathrm{~h}$. Cela reflète les variations entre les races (21).

Par contre, la durée du pic de LH est identique dans les deux lots et s'élève en moyenne à $10,7 \pm 4,1 \mathrm{~h}$. Ce résultat est comparable à celui de Thimonier (21) et Rawlings et al. (17) qui trouvent que la décharge de LH dure de 8 à $12 \mathrm{~h}$. De même, il est conforme au résultat de Cunnigham et al. (8) qui montrent que la durée du pic de LH est généralement inférieure à $12 \mathrm{~h}$.

Le niveau de base et la valeur maximale du pic de LH ne sont pas influencés par la PMSG. La valeur trouvée dans notre étude est comparable au résultat de Pelletier et al. (15) qui ont constaté que le niveau maximum du pic de LH varie entre 50 et $150 \mathrm{ng} / \mathrm{ml}$.

L'intervalle entre le moment de l'ovulation et le début du pic de LH étant constant d'environ 24 h (7), le moment moyen de l'ovulation après le début de l'œstrus a pu être estimé à 36,4 h et $43,5 \mathrm{~h}$ respectivement pour les brebis du lot à PMSG et du lot sans PMSG. Ainsi, l'ovulation a eu lieu plus tôt chez les brebis ayant reçu de la PMSG que chez celles qui ont reçu les éponges seules. Un résultat comparable a été obtenu par Mauléon (12) qui trouve que l'injection de la PMSG accélère l'ovulation chez les brebis traitées. De même, Signoret et Cognie (18) constatent que l'ovulation est précoce chez les brebis ayant reçu de la PMSG à la fin du traitement au FGA. Par contre, elle se produit dans le même délai aussi bien chez les brebis ayant reçu uniquement du FGA que chez les témoins (chaleurs naturelles) selon les mêmes auteurs.

Le moment optimum pour l'insémination artificielle cervicale chez les brebis étant situé aux alentours de $12 \mathrm{~h}$ avant l'ovulation (9), il serait conseillé d'inséminer les brebis Djallonké aux alentours de $24 \mathrm{~h}$ et $31 \mathrm{~h}$ après le début des chaleurs ou 60 et $75 \mathrm{~h}$ après le retrait des éponges selon qu'elles ont reçu ou non de la PMSG à la fin du traitement FGA.

\section{Conclusion}

Le traitement de synchronisation des cycles sexuels chez la brebis Djallonké a été très efficace chez les femelles traitées ou non avec de la PMSG. La PMSG accélère la venue en chaleur et l'apparition du pic de LH sans modifier leurs durées respectives. Une meilleure synchronisation des ovulations due à la PMSG est importante dans le cadre de l'insémination artificielle effectuée à un moment déterminé après le retrait des éponges. La synchronisation des chaleurs à l'aide uniquement d'éponges vaginales au FGA est suffisante pour la brebis Djallonké qui est non saisonnée et exploitée exclusivement en lutte naturelle pour la production de viande. L'évolution de LH pendant les chaleurs chez la brebis Djallonké présente globalement les mêmes caractéristiques que celle des brebis des régions tempérées.

\section{Remerciements}

Les analyses d'hormones ont été prises en charge par le projet FAO/RAF/88/100, à Banjul (Gambie) et par le Centre de coopération Internationale en Recherche Agronomique pour le Développement (CIRAD). Nous remercions Mme N. Jeanguyot (Laboratoire d'hormonologie, UNCEIA) pour sa contribution aimable et efficace dans la réalisation des dosages hormonaux. Nous remercions tous les chercheurs et techniciens du Département des Ressources Animales qui ont aidé aux observations du comportement sexuel des brebis et à la réalisation des prises de sang.

\section{Bibliographie}

1. ACRITOPOULO-FOURCROY S., PAPPAS V., PECLARIS G., ZERVAS N., 1982. Synchronization of oestrus in ewes with provera sponges/PMSG, prostaglandin F2 $\alpha$ or the prostaglandin analogue ICl 80996 and fertility following natural mating or artificial insémination. Reprod. Nulr. Dév., 22 (2) : 345-354.

2. BERGER Ý., 1979. Bilan de 3 années d'études de la race ovine Djallonké en Côte d'Ivoire. Bouaké, Côte d'Ivoire, CRZ, 14 p. (Note technique $n^{\circ} 8 /$ Zoot.)

3. CIIEMINEAU P., MALPAUX B., PLLLETIER J., DELGADILLO J.A., GUERIN Y., THIMONIER J., 1990. Effets de la lumière et de la température sur la reproduction des petits ruminants. In : Réunion annuelle de l'Association pour 1'Etude de la Reproduction animale, Maisons-Alfort, France, 25 janvier 1990. Maisons-Alfort, France, AERA.

4. COGNIE Y., MARIANA J.C., THIMONIER J., 1970. Etude du moment d'ovulation chez la brebis normale ou traitée par un progestagène associé ou non à une injection de PMSG. Ann. Biol. anim., Bioch., Biophys., 10 : $15-24$

5. COGNIE Y., SACARRAMUZI R.J., 1988. Les techniques physiologiques d'accroissement de la fertilité et de la prolificité chez les ovins. In $11^{\mathrm{e}}$ Congrès international de reproduction animale et d'insémination artificielle, Dublin, Irlande, 26-30 juin 1988. Dublin, Irlande, University College of Dublin, p. 623-636.

6. COGNIE Y., SCHIRAR A., MARTINET J., POULIN N., MIRMAN B., 1984. Activité reproductrice et maîtrise de l'ovulation chez les brebis. In $9^{\mathrm{e}}$ Journées pour la Recherche ovine et caprine, Paris, France, 5-6 décembre 1984. Paris, France, ITOVIC-SPEOC, p. 109-133. 
7. CUMMING I.A., BROWN J.M., BLOCKEY M.A.de, WINFIELD C.G., BAXTER R., GODING J.R., 1971. Constancy of interval between luteinizing hormone release and ovulation in the ewe. J. Reprod. Fertil., 24 : 134135 .

8. CUNNINGHAM N.F., SYMONS A.M., SABA N., 1975. Levels of progesterone, I.H and FSH in the plasma of sheep during the oestrus cycle. $J$ Reprod. Fertil., 45 : 177-180.

9. DZIUK P., 1970. Estimation of optimum time for insemination of gilts and ewes by double-mating at certain times relative to ovulation. J. Reprod. Fertil., 22 : 277-282.

10. GAUTHIER D., 1988. The influence of season and shade on oestrus behaviour, timing of preovulatory $\mathrm{LH}$ surge and the pattern of progesterone in FFPN and créole heifers in tropical climate. Reprod. Nutr. Dévelop., 26 (3) : 767-775.

11. LAND R.B.,1970. A relationship between the duration of oestrus, ovulation rate and litter size of sheep. J. Reprod. Fertil., 23 : 49-53.

12. MAULEON M., 1971. Les cycles sexuels. Elevage Insém. 125 : 13-21.

13. MBAYE M., DIOP P.E.H., WONE A., 1989. Etude du cycle sexuel chez la brebis de race Djallonké. In : Atelier de travail sur le bétail trypanotolérant en Afrique, Harare, Zimbabwe, 4-8 septembre 1989. Banjul, Gambie, FAO/RAF/88/100, p. 52-53.

14. OYEDIJI G. O., AKUSU M. O., EGBUNIKE G. N., 1990. Cumparative studies on the effectiveness of sil-estrus implants, veramix sheep sponges and prostaglandin $\mathrm{F} 2 \alpha$ in synchronizing estrus in West African Dwarf shcep. Theriogenology, 34 (3) : 613-618.

15. PELLETIER J., KANN G., DOLAIS J., ROSSELIN G., 1968. Dosage radioimmunologique de l'hormone lutéinisante plasmatique chez le mouton. Comparaison avec le dosage biologique de LH par la diminution de l'acide ascorbique ovarien et exemple d'application aux mesures de la $\mathrm{LH}$ sanguines chez la brebis. C. R. Acad. Sci. (Paris), série D, 286 : 2352-2354.

16. QUIRKE J.F., 1979. Control of reproduction in adult ewes and ewe lambs, and estimation of reproductive wastage in ewe lambs following treatment with progestagen impregnated sponges and PMSG. Livest. Prod. Sci., $6: 295-305$.

17. RAWLINGS N.C., JEFFCOATE I.A., CURRIE W.D., COOK S.J., 1988. Progesterone and the preovulatory LH surge in the ewe. In : 11 Congrès international de reproduction animale et d'insémination artificielle, Dublin, Irlande, 26-30 juin 1988. Dublin, Irlande, University College of Dublin, vol. $2, n^{\circ} 62$

18. SIGNORET J.P., COGNIE Y.,1975. Determination of the moment of ovulation in ewe and sow. Influence of environment and hormonal treatment. Ann. Biol. anim., Bioch., Biophys., 15 (2) : 205-214.
19. SIGNORET J.P., SALMON I., MOLENAT G., 1984. Contrôle des capacités sexuelles et de la production spermatique des béliers avant la lutte et performances obtenues en race Mérinos d'Arles. In : ge Journées pour la Recherche ovine et caprine, Paris, France, 5-6 décembre 1981. Paris, France, ITOVIC-SPEOC, p. 252-264.

20. THIBIER M., SAUMANDE J., 1975. Oestradiol-17ß, progesterone and $17 \alpha$ hydroxyprogesterone concentrations in jugular veinous plasma in cows prior to and during oestrus. J. Steroid Biochem. 6 : 1433-1437.

21. THIMONIER J., 1979. Hormonal control of oestrus cycle in the ewe (a review). Livest. Produc. Sci., $6: 39-50$.

22. THIMONIER J., MAULEON M., 1969. Variations saisonnières du comportement d'oestrus et des activités ovarienne et hypophysaire chez les ovins. Ann. Biol. anim., Bioch., Biophys., 9 (2) : 233-250.

23. THIMONIER J., PELLETIER J., 1971. Différences génétiques dans la décharge ovulante (LH) chez les brebis de race Ile de France : relation avec le nombre d'ovulations. Ann. Biol. anim., Bioch., Biophys., 11 : 559-567.

TOURE G., MEYER C., KOUASSI A. Apparition of heats and LH preovulatory discharge in Djallonké ewes after heat synchronization with or without PMSG. Revue Elev. Méd. vét. Pays trop., 1995, 48 (4) : 357 361

In order to study PMSG influence on the onset of heats and preovulatory LH peak, oestrus of 80 cyclic ewes were synchronized with vaginal sponges (fluorogestone acetate) during the dry and hot season. These ewes were divided into two groups ( $\mathrm{E}$ and $\mathrm{P}$ ) equal in size. At sponge removal, 14 days after insertion, each ewe from group $P$ received $350 \mathrm{IU}$ of PMSG, while group E did not undergo any treatment (control). Sexual behaviour in all ewes was observed every two hours using a vasectomised ram. One day after sponge removal, blood samples were collected every 2 hours for 4 consecutive days in 5 ewes from each group. These blood samples were used to assay the luteinizing hormone (LH) by RIA. Ewes were in heats earlier in group $\mathbf{P}$ than in group $\mathbf{E}$ ( 37.2 $\pm 13.3 \mathrm{~h}$ vs $43.7 \pm 14.3 \mathrm{~h}$ for the period sponge removal - first signs of heats). However, heat duration $(43.6 \pm 20.8 \mathrm{~h})$ was similar in both groups. LH preovulatory surge appeared sooner in group $\mathrm{P}$ than $\mathrm{E}(12.4 \pm$ $1.5 \mathrm{~h}$ vs $19.5 \pm 7.7 \mathrm{~h}$ after the oestrus). LH peak duration $(10.7 \pm 4.1 \mathrm{~h})$ and its maximum value $(90.5 \pm 36.4 \mathrm{ng} / \mathrm{ml})$ were similar in both groups In general, heat and LH peak characteristics observed in Djallonké ewes seemed close to those previously reported in European breeds. In addition, PMSG hastened the onset of both oestrus and LH peak.

Key words : Sheep - Djallonké ewe - Oestrus synchronization - Oestrus cycle - LH - Côte d'Ivoire. 